\title{
High-throughput and simultaneous quantitative analysis of homocysteine-methionine cycle metabolites and co-factors in blood plasma and cerebrospinal fluid by isotope dilution LC-MS/MS
}

\author{
Seu Ping Guiraud ${ }^{1} \cdot$ Ivan Montoliu $^{1} \cdot$ Laeticia Da Silva $^{1} \cdot$ Loïc Dayon $^{1}$ • \\ Antonio Núñez Galindo ${ }^{1}$ • John Corthésy ${ }^{1}$ • Martin Kussmann ${ }^{1}$. \\ Francois-Pierre Martin ${ }^{1}$
}

Received: 5 August 2016/Revised: 30 August 2016/Accepted: 4 October 2016 /Published online: 18 October 2016 (C) The Author(s) 2016. This article is published with open access at Springerlink.com

\begin{abstract}
The methionine cycle is a key pathway contributing to the regulation of human health, with well-established involvement in cardiovascular diseases and cognitive function. Changes in one-carbon cycle metabolites have also been associated with mild cognitive decline, vascular dementia, and Alzheimer's disease. Today, there is no single analytical method to monitor both metabolites and co-factors of the methionine cycle. To address this limitation, we here report for the first time a new method for the simultaneous quantitation of 17 metabolites in the methionine cycle, which are homocysteic acid, taurine, serine, cysteine, glycine, homocysteine, riboflavin, methionine, pyridoxine, cystathionine, pyridoxamine, $S$-adenosylhomocysteine, $S$ adenosylmethionine, betaine, choline, dimethylglycine, and 5-methyltetrahydrofolic acid. This multianalyte method, developed using ultra-performance liquid chromatography-tandem mass spectrometry (UPLC-MS/MS), provides a highly accurate and precise quantitation of these 17 metabolites for both plasma and cerebrospinal fluid metabolite monitoring. The method requires a simple sample preparation, which, combined with a short chromatographic run time, ensures a high sample throughput. This analytical strategy will thus provide a novel metabolomics approach to be employed in
\end{abstract}

Electronic supplementary material The online version of this article (doi:10.1007/s00216-016-0003-1) contains supplementary material, which is available to authorized users.

Seu Ping Guiraud

seuping.guiraud@rd.nestle.com

1 Nestlé Institute of Health Sciences SA, Campus EPFL, Innovation Park, CH-1015 Lausanne, Switzerland large-scale observational and intervention studies. We expect such a robust method to be particularly relevant for broad and deep molecular phenotyping of individuals in relation to their nutritional requirements, health monitoring, and disease risk management.

Keywords Methionine pathway · One-carbon metabolism . LC-MS/MS $\cdot$ High throughput $\cdot$ Plasma $\cdot$ Cerebrospinal fluid

\section{Introduction}

The coupling of the increased percentage of elderly in the world's population and the incidences of chronic diseases is stimulating renewed interest in understanding the role of genetics, environmental factors, and their interactions with individual susceptibility to disease [1]. However, the determination of nutritional requirements for optimizing metabolism for an individual or population remains challenging due to the complexity of food macro- and micronutrient composition, intersubject variability in physiological responses, environmental, and genetic factors [2]. Over the last two decades, the era of omics technologies has provided an innovative paradigm for exploring physiological and pathological processes through broad and deep biological phenotyping [3]. The methionine cycle is among the many central pathways that contribute to human health. Dysfunction of this pathway has been linked to cardiovascular disease, mild cognitive decline, vascular dementia, and Alzheimer's disease [4]. In addition, cofactors derived from diet are crucial for proper functioning of the methionine cycle. A key product of this pathway, $S$ adenosylmethionine (SAM), is itself a co-factor and substrate for methylation of DNA, protein, and RNA as well as 
biosynthetic reactions of key brain metabolites [5]. Hence, optimizing this pathway through nutrition may protect the brain from damage and reduce the risk of cardiovascular events. Monitoring the activity of this pathway in response to nutrition through metabolomics analysis (i.e., nutritional metabolomics) would generate a more comprehensive understanding of the interplay between host, environment, and nutrient interactions [6]. In particular, mass spectrometry (MS)based metabolomics methods have demonstrated robust, accurate, and precise quantitation of several homocysteine-methionine cycle biomarkers in diverse biological matrices [7-10]. However, no single and high-throughput method currently exists to monitor both metabolites and co-factors in the methionine pathway. Most available analytical methods for methionine pathway metabolites have so far deployed multiple and typically separate chromatographic, derivatization, and/or detection schemes to identify and quantify methionine, sulfur-containing amino acids, homocysteine ( $\mathrm{HCy}$ ) and cysteine, $S$-adenosylmethionine/ $S$-adenosylhomocysteine (SAM/ $\mathrm{SAH}$ ), and B vitamins [11-15]. To address this limitation, we have recently developed a new method for the simultaneous quantitation of 13 metabolites and co-factors from the socalled methionine pathway by liquid chromatography-tandem mass spectrometry (LC-MS/MS) in red blood cells [16]. In the present contribution, we introduce a further development of the method to enable a highly accurate and precise quantitation of 17 metabolites in plasma, including homocysteic acid (HA), taurine, serine, cysteine, glycine, homocysteine, riboflavin, methionine, pyridoxine, cystathionine, pyridoxamine, SAH, SAM, betaine, choline, dimethylglycine (DMG), and 5methyltetrahydrofolic acid (5-MTHF) (Fig. 1). This improved method is also based on a simple sample preparation, which combined to a short chromatographic run time ensures a high sample throughput. This novel analytical strategy was also successfully applied for cerebrospinal fluid (CSF) analysis. Thus, this method provides a novel metabolomics approach for large-scale observational and human intervention studies. We demonstrate its applicability by analyzing plasma and CSF samples from healthy elderly subjects and patients diagnosed with Alzheimer's disease (AD).

\section{Materials and methods}

Materials LC-MS-grade acetonitrile (ACN), LC-grade methanol $(\mathrm{MeOH})$, formic acid, perfluoroheptanoic acid (PFHA), ascorbic acid, sodium hydroxide $(\mathrm{NaOH}) 0.1 \mathrm{M}$, hydrochloric acid $(\mathrm{HCl})$, tris(2-carboxyethyl)phosphine (TCEP), dithiothreitol (DTT), and ammonium acetate $\left(\mathrm{NH}_{4} \mathrm{OAc}\right)$ were purchased from Sigma-Aldrich Chemie GmbH (Buchs, Switzerland). Deionized water $(R>18 \Omega \mathrm{M} / \mathrm{cm}$, TOC $<10 \mathrm{ppb})$ was used throughout the experiments and produced by a Millipore-Q water system (Millipore, Bedford, MA, USA).
$\mathrm{HA}$, taurine, serine, cystine, glycine, homocystine (HCy2), riboflavin, methionine, pyridoxine, cystathionine, $\mathrm{SAH}$, pyridoxamine, SAM, DMG, choline, betaine, 5-MTHF, taurine- ${ }^{13} \mathrm{C}_{2}$, glycine- $\mathrm{d}_{2}$, riboflavin-dioxopyrimidine- ${ }^{13} \mathrm{C}_{4}{ }^{15} \mathrm{~N}_{2}$, and methionine- $d_{3}$ standards were purchased from Sigma-Aldrich Chemie $\mathrm{GmbH}$.

HA- $\mathrm{d}_{4}$, serine- $\mathrm{d}_{3}$, pyridoxine- $\mathrm{d}_{2}$, cystathionine- $\mathrm{d}_{4}$, SAM$\mathrm{d}_{4}$, DMG- $\mathrm{d}_{6}$, choline- $\mathrm{d}_{9}$, and betaine- $\mathrm{d}_{11}$ were purchased from CDN Isotopes (Pointe-Claire, Quebec, Canada). Cystine- $\mathrm{d}_{4}$, homocystine- $\mathrm{d}_{8}$, and pyridoxamine- $\mathrm{d}_{3}$ were purchased from Cambridge Isotopes Laboratories (Andover, MA, USA). SAH- $\mathrm{d}_{4}$ was purchased from Cayman Chemical (Ann Arbor, MI, USA). 5 -MTHF- ${ }^{13} \mathrm{C}_{5}$ was purchased from Merck (Schaffhausen, Switzerland).

Preparation of the individual standard and internal standard solutions Standard solutions were prepared individually in $0.1 \mathrm{M} \mathrm{HCl}$ (or in $10 \mathrm{mmol} / \mathrm{L} \mathrm{NH}_{4} \mathrm{OAc}, 0.1 \mathrm{M} \mathrm{NaOH}$, or in a mixture of $\mathrm{MeOH} / \mathrm{H}_{2} \mathrm{O}$ depending on their solubility). Only 5 MTHF was dissolved in a mixture of $10 \mathrm{mmol} / \mathrm{L} \mathrm{NH4OAc}$ with $10 \%$ ascorbic acid and $2 \%$ DTT in $\mathrm{H}_{2} \mathrm{O}$ to prevent oxidation. The same dilution solvents were used for individual internal standard (IS) stock solutions (Table S1, see Electronic Supplementary Material (ESM)). Standard and IS solutions were stored at $-20^{\circ} \mathrm{C}$ for up to 3 months.

Calibration curves and QC samples Calibration standards were prepared by dilution of standard stock solutions in $\mathrm{ACN} / \mathrm{H}_{2} \mathrm{O}(5 / 95 ; v / v)$ to obtain seven calibration solutions (calibrants 1 to 7 ) in micromolar range for HA, taurine, serine, cystine, glycine, methionine, HCy2, DMG, betaine, and choline and in nanomolar range for riboflavin, pyridoxine, cystathionine, pyridoxamine, SAH, SAM, and 5-MTHF as described in Table S2 (see ESM). An IS working solution was prepared by dilution of IS standard stock solutions in ACN/ $\mathrm{H}_{2} \mathrm{O}(5 / 95 ; v / v)$ (see ESM Table S3) and stored at $-20{ }^{\circ} \mathrm{C}$ for up to 3 months. Calibration samples were prepared with each batch of samples and injected at both the beginning and the end of the sample sequence. Fifty microliters of each calibration solution was pipetted and transferred into a $1.5-\mathrm{mL}$ microcentrifuge tube. A volume of 10 microliters of IS working solution was then added to these solutions. Fifty microliters of TCEP $(100 \mathrm{mg} / \mathrm{mL})$ and then $140 \mu \mathrm{L}$ of $\mathrm{ACN} / \mathrm{H}_{2} \mathrm{O}(5 / 95 ; v / v)$ solution were furtherly added to the mixture. The tubes were placed on an autosampler rack and vortexed on a plate vortexer for $15 \mathrm{~min}$ at $1350 \mathrm{rpm}$. The solutions were transferred into vials and ready for LC-MS/MS analysis. Quality controls (QCs) at two different concentrations were added to each batch of samples. The low QC (at the same concentration as calibration level 3) and high QC (calibration level 6) samples were prepared using the same protocol as the calibration samples. 
Fig. 1 Metabolic pathway involving the measured (blue) metabolites from the homocysteine-methionine cycle

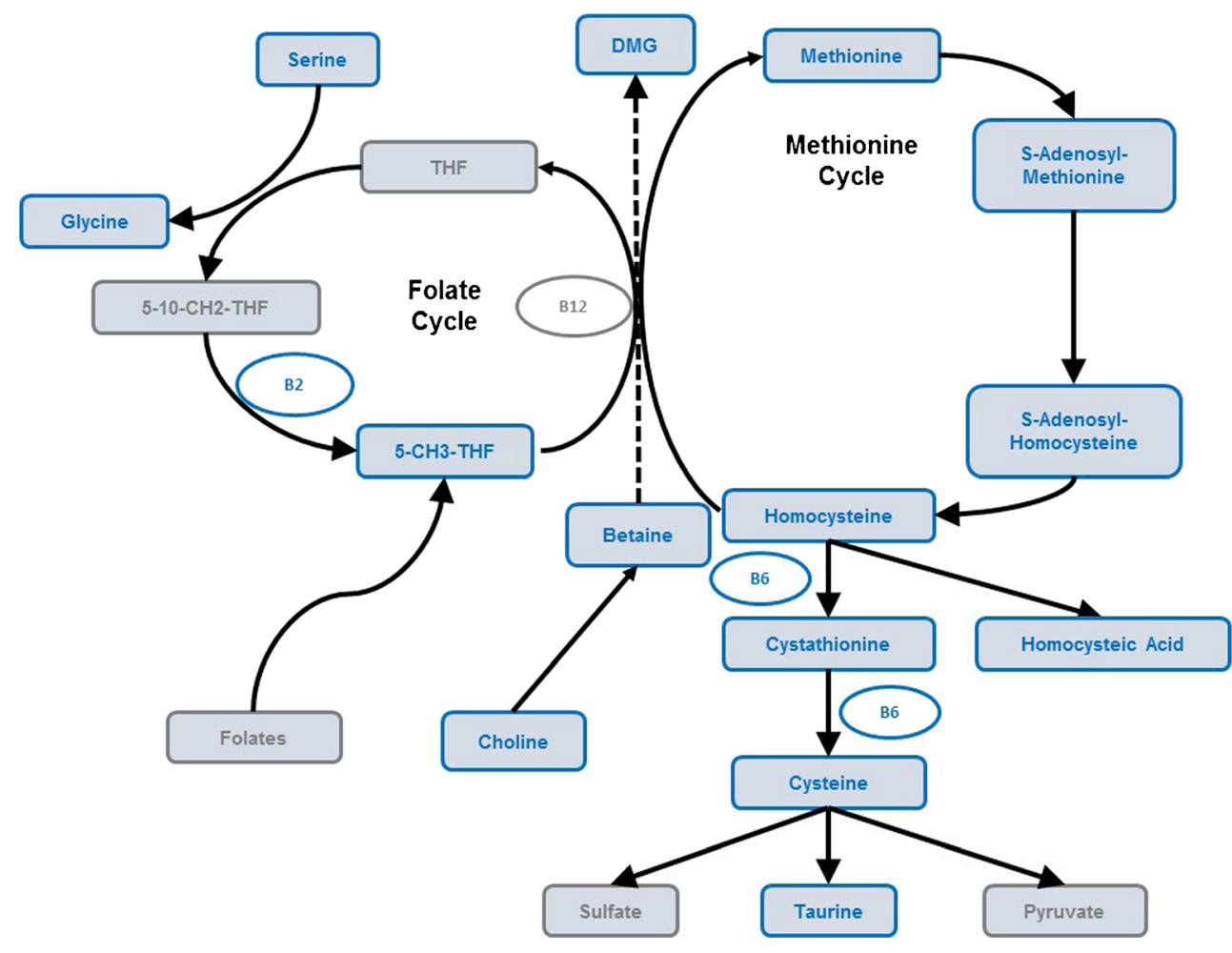

LC-MS/MS instrumentation The instrumental methodology was previously reported for red blood cell analysis [16] and is fully described in the ESM (Table S4 and Table S5). Briefly, a high-throughput method was developed using LC-MS/MS. Separation and analysis were performed on an Accela UHPLC 1250 Pump (Thermo Fisher Scientific Inc., Waltham, MA, USA) coupled to a TSQ Quantum Vantage triple quadrupole (Thermo Fisher Scientific Inc., Waltham, MA, USA) equipped with a heated electrospray ionization (H-ESI) source. Chromatographic separation was obtained using gradient elution on a reversed-phase UPLC XSelect HSST3 $2.5 \mu \mathrm{m}, 100 \times 2.1 \mathrm{~mm}$ I.D. column (Waters Corporation, Milford, MA, USA). The injection volume was $10 \mu \mathrm{L}$ and the total run time of analysis was $13 \mathrm{~min}$.

Human sample collection for method validation The human sub-cohort was supplied from PrecisionMed, Inc. (CA, USA, protocol 8009). This sub-cohort comprises 12 individuals: 6 control subjects and 6 patients diagnosed with Alzheimer's disease (AD). All the subjects are aged 50 years and older.

Plasma and CSF sample collection and processing Plasma or CSF frozen samples were thawed and vortexed for $10 \mathrm{~s}$. A $50-\mu \mathrm{L}$ volume of plasma or CSF samples was transferred by pipetting into a $1.5-\mathrm{mL}$ microcentrifuge tube. Ten microliters of IS solution was added to plasma or CSF samples. Fifty microliters of TCEP solution and then $140 \mu \mathrm{L}$ of methanol + $1 \%$ FA were then added to the mixture. The tubes were placed in a multitube vortexer for $15 \mathrm{~min}$ at $1350 \mathrm{rpm}$ at $4{ }^{\circ} \mathrm{C}$ and centrifuged at $14,500 \mathrm{rpm}$ for $5 \mathrm{~min}$. The supernatants were pipetted and filtered through a $0.22-\mu \mathrm{m}$ filter and placed into vials for LC-MS/MS analysis.

Matrix effects Matrix effects were assessed by considering the post-extraction IS analyte spiking. Two sets of samples (five replicates for each set) were used: one containing the IS analyte added to an extracted matrix (post-extraction sample) and the other containing the IS analyte in the mobile phase solvent. Both sets of samples were spiked with the same concentration of IS analyte. Matrix effect values in percent were calculated using the following equation: matrix effect $(\%)=B / A * 100(A=$ mean of external solution peak area, $B=$ post-extraction sample peak area).

Validation procedure The validation procedure involves the following criteria: specificity and selectivity, limit of detection (LOD) and limit of quantitation (LOQ), linearity, trueness, precision (repeatability and intermediate precision), and recovery. Specificity and selectivity were assessed using the retention time and mass spectrum profile of pure standards and compared to the ones in unspiked CSF and plasma samples. 

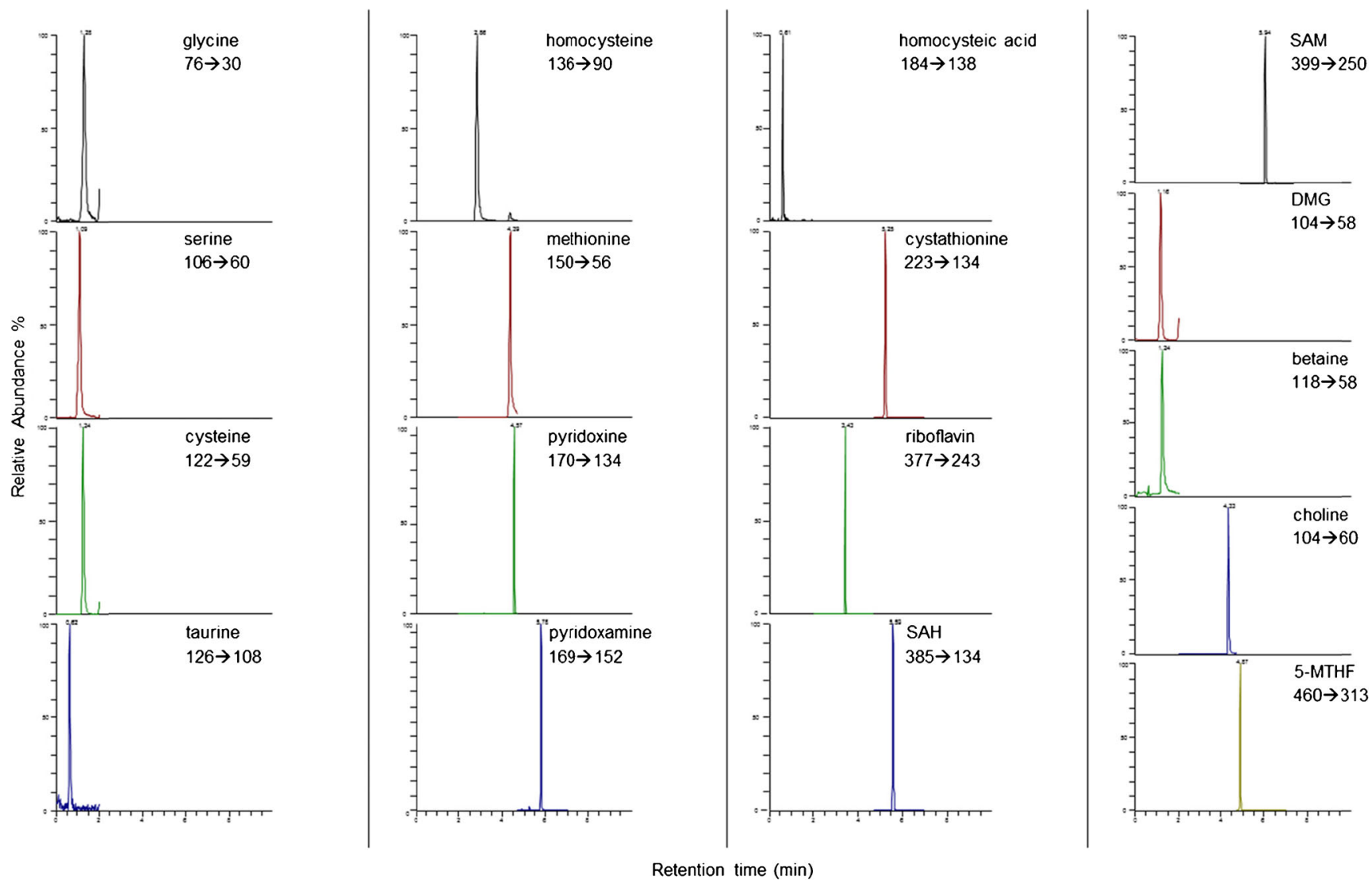

Fig. 2 Typical SRM chromatograms obtained for spiked CSF samples at the medium level

LOD and LOQ were evaluated based on the signal-to-noise ratio $(S / N)$, at least above 3 for the LOD and 10 for the LOQ by injecting spiked plasma or CSF samples with the IS mixture of all analytes.

The calibration curves were constructed, at each concentration level, by calculating the chromatographic peak area ratio of the analyte and its IS for each metabolite.

Trueness and precision (repeatability and intermediate precision) were assessed by spiking plasma and CSF samples at low, medium, and high levels in six replicates for each level. For repeatability, six replicates were performed by the same operator on three separate occasions in a short period of time (less than 1 month). For intermediate precision, the same protocol was followed with additional analyses being carried out by two other individuals on a total of six separated occasions.

Statistical analysis Two-sample Kolmogorov-Smirnov (KS) tests were used to determine the statistical significance between gender distributions of metabolites. Uncorrected $P$ values less than 0.05 were considered significant. The statistical software R v.3.2.2 [17] was used as a general platform for such analyses, and the package ggplot2 [18] was used for visualization.

\section{Results and discussion}

The analytical method analyzes 17 metabolites involved in the homocysteine-methionine cycle. The previously described instrumental methodology used for the RBC matrix [16] was applied to the analysis of plasma and CSF samples. The analytical method extends the metabolite coverage, which now also includes betaine, DMG, choline, and 5-MTHF acid. Such an approach enables a more comprehensive assessment of homocysteine contribution via methionine synthase but also through betaine homocysteine methyltransferase (BHMT) to the methionine metabolism. The BHMT pathway is particularly active in both liver and kidney, which are the main organs for storage of large amounts of betaine [19]. This metabolite is actually distributed widely in plants and animals, particularly in seafood, and an inadequate dietary intake leads to hypomethylation of proteins, RNA, and DNA. Inadequate levels of betaine lead to disturbed hepatic protein metabolism, expressed by elevated homocysteine concentrations and decreased SAM concentrations in plasma [20]. Less well known are the correlations between betaine availability in plasma and its concentration in the CSF. Changes in the methionine cycle may be monitored by betaine-DMG conversion and the variations in 5-MTHF concentrations in plasma. In addition to betaine, choline is another important dietary methyl group 
Table 1 Single reaction monitoring (SRM) transitions

\begin{tabular}{|c|c|c|c|}
\hline Analyte & $\mathrm{RT}^{\mathrm{a}}(\min )$ & $\begin{array}{l}\text { SRM transitions } \\
(m / z)\end{array}$ & $\mathrm{CE}(\mathrm{eV})$ \\
\hline Glycine & 1.4 & $76 \rightarrow 30$ & 11 \\
\hline Glycine- $\mathrm{d}_{2}$ & 1.4 & $78 \rightarrow 32$ & 7 \\
\hline Serine & 1.15 & $106 \rightarrow 60$ & 10 \\
\hline Serine- $d_{3}$ & 1.15 & $109 \rightarrow 63$ & 11 \\
\hline Cysteine & 1.4 & $122 \rightarrow 59$ & 20 \\
\hline Cysteine- $\mathrm{d}_{2}$ & 1.4 & $124 \rightarrow 61$ & 23 \\
\hline Taurine & 0.6 & $126 \rightarrow 108$ & 11 \\
\hline Taurine- ${ }^{13} \mathrm{C}_{2}$ & 0.6 & $128 \rightarrow 110$ & 11 \\
\hline Homocysteine & 2.85 & $136 \rightarrow 90$ & 10 \\
\hline Homocysteine- $\mathrm{d}_{4}$ & 2.85 & $140 \rightarrow 94$ & 10 \\
\hline Methionine & 4.3 & $150 \rightarrow 104$ & 10 \\
\hline Methionine- $\mathrm{d}_{3}$ & 4.3 & $153 \rightarrow 107$ & 9 \\
\hline Pyridoxine & 4.5 & $170 \rightarrow 134$ & 18 \\
\hline Pyridoxine- $\mathrm{d}_{2}$ & 4.5 & $172 \rightarrow 136$ & 19 \\
\hline Pyridoxamine & 5.75 & $169 \rightarrow 152$ & 11 \\
\hline Pyridoxamine- $\mathrm{d}_{3}$ & 5.75 & $172 \rightarrow 155$ & 12 \\
\hline Homocysteic acid & 0.6 & $184 \rightarrow 138$ & 10 \\
\hline Homocysteic acid- $\mathrm{d}_{4}$ & 0.6 & $188 \rightarrow 142$ & 9 \\
\hline Cystathionine & 5.2 & $223 \rightarrow 134$ & 13 \\
\hline Cystathionine- $\mathrm{d}_{4}$ & 5.2 & $227 \rightarrow 138$ & 13 \\
\hline Riboflavin & 3.4 & $377 \rightarrow 243$ & 15 \\
\hline Riboflavin $-{ }^{13} \mathrm{C}_{4}{ }^{15} \mathrm{~N}_{2}$ & 3.4 & $383 \rightarrow 249$ & 13 \\
\hline $\mathrm{SAH}$ & 5.6 & $385 \rightarrow 134$ & 21 \\
\hline $\mathrm{SAH}-\mathrm{d}_{4}$ & 5.6 & $389 \rightarrow 136$ & 18 \\
\hline SAM & 5.9 & $399 \rightarrow 250$ & 14 \\
\hline SAM-d ${ }_{3}$ & 5.9 & $402 \rightarrow 250$ & 13 \\
\hline DMG & 1.25 & $104 \rightarrow 58$ & 12 \\
\hline DMG-d $_{6}$ & 1.25 & $110 \rightarrow 64$ & 15 \\
\hline Betaine & 1.4 & $118 \rightarrow 58$ & 25 \\
\hline Betaine- $d_{11}$ & 1.4 & $129 \rightarrow 66$ & 31 \\
\hline Choline & 4.35 & $104 \rightarrow 60$ & 17 \\
\hline Choline-d, & 4.35 & $113 \rightarrow 69$ & 19 \\
\hline 5-MTHF & 4.9 & $460 \rightarrow 313$ & 15 \\
\hline 5-MTHF- ${ }^{13} \mathrm{C}_{5}$ & 4.9 & $465 \rightarrow 313$ & 26 \\
\hline
\end{tabular}

${ }^{\mathrm{a}} \mathrm{RT}$ retention time given as information only

donor particularly during folate deficiency [21]. Choline may enter the homocysteine-methionine cycle through its oxidation to betaine which is used as a substrate in the BHMT reaction that links choline and betaine to the folatedependent methionine pathway. Our analytical method combines LC with MS/MS which provides a high specificity and sensitivity, as well as a fast run time (13 min). Compared to our previous methodology for RBC analysis, the current workflow for plasma and CSF does not require the same sample preparation, since there is no metabolite extraction from
Table 2 Estimated limits of detection (LOD) and limits of quantitation (LOQ) values from plasma and CSF samples spiked with labeled internal standard (IS) solutions

\begin{tabular}{|c|c|c|c|c|c|c|}
\hline \multirow[t]{2}{*}{ Analyte } & \multicolumn{3}{|c|}{ In plasma } & \multicolumn{3}{|c|}{ In CSF } \\
\hline & LOD & LOQ & Units & LOD & LOQ & Units \\
\hline Homocysteic acid & 0.5 & 1.0 & $\mu \mathrm{M}$ & 0.25 & 0.5 & $\mu \mathrm{M}$ \\
\hline Taurine & 2.0 & 3.0 & $\mu \mathrm{M}$ & 4.0 & 5.0 & $\mu \mathrm{M}$ \\
\hline Serine & 2.0 & 3.0 & $\mu \mathrm{M}$ & 0.5 & 1.0 & $\mu \mathrm{M}$ \\
\hline Total cysteine & 0.1 & 0.5 & $\mu \mathrm{M}$ & 0.05 & 0.125 & $\mu \mathrm{M}$ \\
\hline Glycine & 2.0 & 3.0 & $\mu \mathrm{M}$ & 1.0 & 2.0 & $\mu \mathrm{M}$ \\
\hline Total homocysteine & 1.0 & 2.0 & $\mu \mathrm{M}$ & 0.125 & 0.25 & $\mu \mathrm{M}$ \\
\hline Riboflavin & 1.0 & 2.0 & $\mathrm{nM}$ & 4.0 & 5.0 & $\mathrm{nM}$ \\
\hline Methionine & 2.0 & 3.0 & $\mu \mathrm{M}$ & 0.5 & 1.0 & $\mu \mathrm{M}$ \\
\hline Pyridoxine & 2.0 & 3.0 & $\mathrm{nM}$ & 1.5 & 2.0 & $\mathrm{nM}$ \\
\hline Cystathionine & 0.5 & 1.0 & $\mathrm{nM}$ & 0.5 & 1.0 & $\mathrm{nM}$ \\
\hline SAH & 0.1 & 0.5 & $\mathrm{nM}$ & 2.0 & 4.0 & $\mathrm{nM}$ \\
\hline Pyridoxamine & 2.0 & 3.0 & $\mathrm{nM}$ & 0.25 & 0.5 & $\mathrm{nM}$ \\
\hline SAM & 2.0 & 3.0 & $\mathrm{nM}$ & 2.0 & 4.0 & $\mathrm{nM}$ \\
\hline DMG & 2.0 & 4.0 & $\mu \mathrm{M}$ & 0.1 & 0.4 & $\mu \mathrm{M}$ \\
\hline Betaine & 0.05 & 0.125 & $\mu \mathrm{M}$ & 0.01 & 0.02 & $\mu \mathrm{M}$ \\
\hline Choline & 0.02 & 0.05 & $\mu \mathrm{M}$ & 0.05 & 0.1 & $\mu \mathrm{M}$ \\
\hline 5-MTHF & 6.0 & 8.0 & $\mathrm{nM}$ & 2.0 & 4.0 & $\mathrm{nM}$ \\
\hline
\end{tabular}

cells. Sample treatment was optimized for plasma samples and then applied to CSF samples. A simple and quick sample preparation including protein precipitation and chemical reduction was required for total cysteine and total homocysteine quantitation.

Extraction method development Homocysteine is found primarily in plasma in the form of free homocysteine (HCy), dimers, and analogue molecular forms bound by disulfide bridges to proteins or other thiol-containing compounds. The presence of these multiple forms complicates the development of reliable analytical methods. One common approach consists in the chemical reduction of all disulfide bonds to provide a total homocysteine measurement. Total homocysteine is therefore the sum of all homocysteine obtained from the reduction of these disulfide bonds; this also applies to total cysteine. Two reducing agents are frequently used for the reduction of disulfide bounds: DTT [22-26] and TCEP [27, 28]. We assessed both reducing agents and found that TCEP performed best in terms of stability and extraction efficiency. Indeed, TCEP provides a rapid chemical reduction of disulfide bonds at room temperature without any $\mathrm{pH}$ adjustments [29]. Yet, a concentration of $200 \mathrm{mM}$ TCEP was found not sufficient to totally reduce the disulfide bonds, and the concentration was increased to $100 \mathrm{mg} / \mathrm{mL}$ [30]. Common solvents such as $\mathrm{MeOH}$ and $\mathrm{ACN}$ [31] are reported for protein precipitation for the analysis of total homocysteine in blood with or 
Table 3 Repeatability and intermediate trueness and precision values at three different concentration levels $(n=6$ per day, per level) to determine accuracy of the developed method in plasma samples

\begin{tabular}{|c|c|c|c|c|c|c|}
\hline Analyte & $\begin{array}{l}\text { Targeted } \\
\text { concentration }\end{array}$ & Mean \pm SD & Units & $\begin{array}{l}\text { Recovery } \\
\pm \operatorname{RSD}(\%)\end{array}$ & $\mathrm{CV}_{r}(\%)$ & $\mathrm{CV}_{\mathrm{iR}}(\%)$ \\
\hline \multirow[t]{3}{*}{ Homocysteic acid } & 15.0 & $15.0 \pm 0.8$ & \multirow[t]{3}{*}{$\mu \mathrm{M}$} & $100.2 \pm 2.1$ & 6.2 & 7.7 \\
\hline & 100.0 & $102.2 \pm 3.9$ & & $102.2 \pm 1.6$ & 6.6 & 7.2 \\
\hline & 200.0 & $199.6 \pm 7.3$ & & $99.8 \pm 1.5$ & 6.3 & 6.8 \\
\hline \multirow[t]{3}{*}{ Taurine } & 30.0 & $29.6 \pm 2.8$ & \multirow[t]{3}{*}{$\mu \mathrm{M}$} & $98.6 \pm 3.8$ & 12.5 & 14.7 \\
\hline & 200.0 & $188.0 \pm 14.3$ & & $94.0 \pm 3.1$ & 7.3 & 10.1 \\
\hline & 400.0 & $370.0 \pm 24.0$ & & $92.5 \pm 4.8$ & 5.7 & 8.3 \\
\hline \multirow[t]{3}{*}{ Serine } & 30.0 & $30.1 \pm 0.8$ & \multirow[t]{3}{*}{$\mu \mathrm{M}$} & $100.3 \pm 1.1$ & 7.9 & 7.7 \\
\hline & 200.0 & $203.4 \pm 6.2$ & & $101.7 \pm 1.2$ & 3.2 & 4.3 \\
\hline & 400.0 & $405.6 \pm 11.6$ & & $101.4 \pm 1.2$ & 4.1 & 4.7 \\
\hline \multirow[t]{3}{*}{ Total cysteine } & 15.0 & $16.1 \pm 0.9$ & \multirow[t]{3}{*}{$\mu \mathrm{M}$} & $107.6 \pm 4.1$ & 14.1 & 14.0 \\
\hline & 100.0 & $101.5 \pm 1.9$ & & $101.5 \pm 0.8$ & 7.9 & 7.5 \\
\hline & 200.0 & $191.4 \pm 12.5$ & & $95.7 \pm 2.7$ & 7.1 & 9.2 \\
\hline \multirow[t]{3}{*}{ Glycine } & 30.0 & $28.6 \pm 1.8$ & \multirow[t]{3}{*}{$\mu \mathrm{M}$} & $95.3 \pm 2.6$ & 15.2 & 15.2 \\
\hline & 200.0 & $197.9 \pm 6.1$ & & $99.0 \pm 1.3$ & 5.1 & 5.6 \\
\hline & 400.0 & $399.9 \pm 15.8$ & & $100.0 \pm 1.6$ & 4.8 & 5.9 \\
\hline \multirow[t]{3}{*}{ Total homocysteine } & 15.0 & $14.6 \pm 0.4$ & \multirow[t]{3}{*}{$\mu \mathrm{M}$} & $97.5 \pm 1.0$ & 2.9 & 3.7 \\
\hline & 100.0 & $100.3 \pm 1.7$ & & $100.3 \pm 0.7$ & 2.2 & 2.6 \\
\hline & 200.0 & $197.0 \pm 5.0$ & & $98.5 \pm 1.0$ & 6.2 & 6.2 \\
\hline \multirow[t]{3}{*}{ Riboflavin } & 30.0 & $29.5 \pm 3.1$ & \multirow[t]{3}{*}{$\mathrm{nM}$} & $98.3 \pm 4.3$ & 18.0 & 19.5 \\
\hline & 200.0 & $193.9 \pm 10.2$ & & $97.0 \pm 2.1$ & 6.2 & 7.7 \\
\hline & 400.0 & $399.5 \pm 28.0$ & & $99.9 \pm 2.9$ & 8.6 & 10.5 \\
\hline \multirow[t]{3}{*}{ Methionine } & 15.0 & $15.2 \pm 0.4$ & \multirow[t]{3}{*}{$\mu \mathrm{M}$} & $101.4 \pm 1.1$ & 6.1 & 6.2 \\
\hline & 100.0 & $103.1 \pm 2.4$ & & $103.1 \pm 1.8$ & 2.8 & 3.5 \\
\hline & 200.0 & $202.7 \pm 5.9$ & & $101.4 \pm 1.2$ & 3.6 & 4.4 \\
\hline \multirow[t]{3}{*}{ Pyridoxine } & 15.0 & $14.4 \pm 0.4$ & \multirow[t]{3}{*}{$\mathrm{nM}$} & $96.0 \pm 2.4$ & 9.1 & 8.8 \\
\hline & 100.0 & $100.8 \pm 3.2$ & & $100.8 \pm 1.3$ & 4.0 & 4.8 \\
\hline & 200.0 & $194.8 \pm 9.3$ & & $97.4 \pm 1.9$ & 5.2 & 6.7 \\
\hline \multirow[t]{3}{*}{ Cystathionine } & 30.0 & $30.8 \pm 1.9$ & \multirow[t]{3}{*}{$\mathrm{nM}$} & $102.5 \pm 2.5$ & 17.2 & 16.8 \\
\hline & 200.0 & $194.7 \pm 16.8$ & & $97.3 \pm 3.5$ & 9.7 & 12.4 \\
\hline & 400.0 & $388.3 \pm 26.5$ & & $97.1 \pm 2.8$ & 8.6 & 10.4 \\
\hline SAH & 30.0 & $30.8 \pm 3.1$ & $\mathrm{nM}$ & $102.6 \pm 4.1$ & 17.9 & 19.1 \\
\hline & 200.0 & $202.2 \pm 11.2$ & & $101.1 \pm 2.3$ & 12.7 & 12.8 \\
\hline & 400.0 & $398.8 \pm 25.0$ & & $99.7 \pm 2.6$ & 11.7 & 12.4 \\
\hline Pyridoxamine & 15.0 & $13.5 \pm 1.0$ & $\mathrm{nM}$ & $90.3 \pm 6.2$ & 16.0 & 16.4 \\
\hline & 100.0 & $98.5 \pm 4.4$ & & $98.5 \pm 1.8$ & 7.5 & 8.2 \\
\hline & 200.0 & $195.1 \pm 11.3$ & & $97.5 \pm 2.4$ & 9.5 & 10.4 \\
\hline SAM & 30.0 & $26.5 \pm 2.0$ & $\mathrm{nM}$ & $88.4 \pm 7.2$ & 18.5 & 18.5 \\
\hline & 200.0 & $200.6 \pm 10.0$ & & $100.3 \pm 2.0$ & 12.1 & 12.1 \\
\hline & 400.0 & $415.9 \pm 36.7$ & & $104.0 \pm 3.6$ & 10.1 & 12.7 \\
\hline DMG & 5.0 & $4.9 \pm 0.1$ & $\mu \mathrm{M}$ & $97.6 \pm 1.4$ & 5.2 & 5.1 \\
\hline & 40.0 & $39.5 \pm 2.0$ & & $98.7 \pm 2.1$ & 3.9 & 6.2 \\
\hline & 80.0 & $79.5 \pm 2.2$ & & $99.3 \pm 1.1$ & 2.4 & 3.6 \\
\hline Betaine & 12.5 & $12.6 \pm 0.9$ & $\mu \mathrm{M}$ & $100.7 \pm 2.9$ & 7.0 & 9.5 \\
\hline & 100.0 & $96.3 \pm 2.3$ & & $96.3 \pm 2.1$ & 4.9 & 5.1 \\
\hline & 200.0 & $196.3 \pm 5.0$ & & $98.1 \pm 1.0$ & 3.0 & 3.7 \\
\hline Choline & 5.0 & $4.9 \pm 0.2$ & $\mu \mathrm{M}$ & $98.8 \pm 1.3$ & 5.3 & 5.8 \\
\hline & 40.0 & $39.4 \pm 0.8$ & & $98.4 \pm 0.8$ & 4.2 & 4.3 \\
\hline & 80.0 & $79.4 \pm 1.7$ & & $99.2 \pm 0.9$ & 1.4 & 2.5 \\
\hline 5-MTHF & 50.0 & $47.8 \pm 2.7$ & $\mathrm{nM}$ & $95.7 \pm 2.3$ & 15.1 & 14.9 \\
\hline & 400.0 & $393.0 \pm 26.5$ & & $98.3 \pm 2.7$ & 12.8 & 13.5 \\
\hline & 800.0 & $804.4 \pm 26.8$ & & $100.5 \pm 1.4$ & 10.5 & 10.2 \\
\hline
\end{tabular}

without the addition of acids such as FA. We found that $\mathrm{MeOH}+1 \% \mathrm{FA}$ gave on overall the best mass spectral peak intensities and shapes.

Chromatography and mass spectra The chromatographic method optimization is fully described in our previously published paper [16]. In this method, retention of the most polar compounds on a reversed-phase column was not possible without the use of ion-pairing agents. Typical chromatograms of a spiked CSF sample at the medium level are shown in Fig. 2. Optimal selected reaction monitoring (SRM) conditions were obtained in positive electrospray ionization mode and are reported in Table 1.

Validation procedures. Estimated LOD and LOQ Due to endogenous levels of some analytes and incomplete reference material for all the analyzed metabolites, the LOD and LOQ were estimated by injecting plasma and CSF 
Table 4 Repeatability and intermediate trueness and precision values at three different concentration levels $(n=6$ per day, per level) to determine accuracy of the developed method in CSF samples

\begin{tabular}{|c|c|c|c|c|c|c|}
\hline Analyte & $\begin{array}{l}\text { Targeted } \\
\text { concentration }\end{array}$ & Mean \pm SD & Units & $\begin{array}{l}\text { Recovery } \\
\pm \operatorname{RSD}(\%)\end{array}$ & $\mathrm{CV}_{r}(\%)$ & $\mathrm{CV}_{\mathrm{iR}}(\%)$ \\
\hline \multirow[t]{3}{*}{ Homocysteic acid } & 12.5 & $12.9 \pm 0.3$ & \multirow[t]{3}{*}{$\mu \mathrm{M}$} & $103.2 \pm 1.8$ & 6.7 & 6.5 \\
\hline & 100.0 & $102.4 \pm 4.2$ & & $102.4 \pm 1.7$ & 4.8 & 6.0 \\
\hline & 200.0 & $200.7 \pm 8.5$ & & $100.4 \pm 1.7$ & 3.9 & 5.5 \\
\hline \multirow[t]{3}{*}{ Taurine } & 25.0 & $25.4 \pm 0.5$ & \multirow[t]{3}{*}{$\mu \mathrm{M}$} & $101.7 \pm 0.9$ & 8.8 & 8.3 \\
\hline & 200.0 & $199.4 \pm 10.6$ & & $99.7 \pm 2.2$ & 5.0 & 7.0 \\
\hline & 400.0 & $386.5 \pm 16.7$ & & $96.6 \pm 1.8$ & 3.1 & 5.2 \\
\hline \multirow[t]{3}{*}{ Serine } & 25.0 & $24.5 \pm 1.1$ & \multirow[t]{3}{*}{$\mu \mathrm{M}$} & $98.1 \pm 1.7$ & 6.5 & 7.3 \\
\hline & 200.0 & $202.6 \pm 5.9$ & & $101.3 \pm 1.2$ & 3.8 & 4.6 \\
\hline & 400.0 & $402.8 \pm 14.3$ & & $100.7 \pm 1.4$ & 2.7 & 4.3 \\
\hline \multirow[t]{3}{*}{ Total cysteine } & 12.5 & $12.7 \pm 0.4$ & \multirow[t]{3}{*}{$\mu \mathrm{M}$} & $101.3 \pm 1.4$ & 4.7 & 5.4 \\
\hline & 100.0 & $102.8 \pm 3.0$ & & $102.7 \pm 1.2$ & 3.2 & 4.2 \\
\hline & 200.0 & $201.0 \pm 4.9$ & & $100.5 \pm 1.0$ & 2.7 & 3.5 \\
\hline \multirow[t]{3}{*}{ Glycine } & 25.0 & $25.2 \pm 1.3$ & \multirow[t]{3}{*}{$\mu \mathrm{M}$} & $100.7 \pm 2.1$ & 5.3 & 7.0 \\
\hline & 200.0 & $201.7 \pm 6.0$ & & $100.8 \pm 1.2$ & 3.4 & 4.3 \\
\hline & 400.0 & $393.3 \pm 8.3$ & & $98.3 \pm 0.9$ & 2.5 & 3.1 \\
\hline \multirow[t]{3}{*}{ Total homocysteine } & 12.5 & $12.4 \pm 0.6$ & \multirow[t]{3}{*}{$\mu \mathrm{M}$} & $99.2 \pm 1.9$ & 2.6 & 5.3 \\
\hline & 100.0 & $102.7 \pm 1.8$ & & $102.7 \pm 1.5$ & 3.5 & 3.7 \\
\hline & 200.0 & $202.4 \pm 4.4$ & & $101.2 \pm 0.9$ & 2.2 & 2.9 \\
\hline \multirow[t]{3}{*}{ Riboflavin } & 25.0 & $25.4 \pm 2.8$ & \multirow[t]{3}{*}{$\mathrm{nM}$} & $101.7 \pm 4.5$ & 16 & 18.3 \\
\hline & 200.0 & $202.1 \pm 7.8$ & & $101.0 \pm 1.6$ & 11.7 & 11.4 \\
\hline & 400.0 & $402.5 \pm 23.8$ & & $100.6 \pm 2.4$ & 7.8 & 9.2 \\
\hline \multirow[t]{3}{*}{ Methionine } & 12.5 & $12.9 \pm 0.6$ & \multirow[t]{3}{*}{$\mu \mathrm{M}$} & $103.0 \pm 1.8$ & 4.9 & 6.3 \\
\hline & 100.0 & $104.2 \pm 4.2$ & & $104.2 \pm 1.6$ & 3.4 & 5.1 \\
\hline & 200.0 & $203.9 \pm 6.5$ & & $102.0 \pm 1.3$ & 3.4 & 4.5 \\
\hline \multirow[t]{3}{*}{ Pyridoxine } & 12.5 & $12.6 \pm 0.3$ & \multirow[t]{3}{*}{$\mathrm{nM}$} & $100.9 \pm 0.9$ & 6.6 & 6.4 \\
\hline & 100.0 & $102.3 \pm 4.3$ & & $102.3 \pm 1.7$ & 3.9 & 5.5 \\
\hline & 200.0 & $200.7 \pm 6.8$ & & $100.4 \pm 1.4$ & 3.3 & 4.5 \\
\hline \multirow[t]{3}{*}{ Cystathionine } & 25.0 & $24.1 \pm 1.0$ & \multirow[t]{3}{*}{$\mathrm{nM}$} & $96.5 \pm 1.6$ & 11.4 & 11.1 \\
\hline & 200.0 & $213.9 \pm 16.3$ & & $107.0 \pm 3.1$ & 7.2 & 10.1 \\
\hline & 400.0 & $433.1 \pm 28.4$ & & $108.3 \pm 4.7$ & 7.1 & 9.2 \\
\hline SAH & 50.0 & $48.1 \pm 3.5$ & $\mathrm{nM}$ & $96.3 \pm 3.0$ & 12.8 & 13.8 \\
\hline & 400.0 & $403.8 \pm 14.3$ & & $100.9 \pm 1.4$ & 6.9 & 7.2 \\
\hline & 800.0 & $773.0 \pm 18.5$ & & $96.6 \pm 2.0$ & 6.9 & 6.7 \\
\hline Pyridoxamine & 12.5 & $12.1 \pm 0.4$ & $\mathrm{nM}$ & $96.8 \pm 2.0$ & 9.9 & 9.5 \\
\hline & 100.0 & $101.3 \pm 3.5$ & & $101.3 \pm 1.4$ & 7.8 & 7.9 \\
\hline & 200.0 & $200.0 \pm 8.8$ & & $100.0 \pm 1.8$ & 7.6 & 8.2 \\
\hline SAM & 50.0 & $51.1 \pm 4.4$ & $\mathrm{nM}$ & $102.1 \pm 3.5$ & 13.9 & 15.3 \\
\hline & 400.0 & $406.2 \pm 19.1$ & & $101.6 \pm 1.9$ & 8.5 & 9.1 \\
\hline & 800.0 & $807.2 \pm 41.6$ & & $100.9 \pm 2.1$ & 5.9 & 7.5 \\
\hline DMG & 5.0 & $5.0 \pm 0.2$ & $\mu \mathrm{M}$ & $99.8 \pm 1.2$ & 3.1 & 4.1 \\
\hline & 40.0 & $40.6 \pm 1.1$ & & $101.5 \pm 1.1$ & 3.2 & 4.0 \\
\hline & 80.0 & $80.8 \pm 2.1$ & & $101.0 \pm 1.0$ & 2.8 & 3.6 \\
\hline Betaine & 5.0 & $5.2 \pm 0.2$ & $\mu \mathrm{M}$ & $102.5 \pm 1.5$ & 5.2 & 6.1 \\
\hline & 40.0 & $41.1 \pm 1.0$ & & $102.8 \pm 1.7$ & 3.2 & 3.8 \\
\hline & 80.0 & $80.5 \pm 2.6$ & & $100.7 \pm 1.3$ & 2.3 & 3.8 \\
\hline Choline & 5.0 & $5.0 \pm 0.1$ & $\mu \mathrm{M}$ & $100.2 \pm 1.2$ & 4.9 & 5.3 \\
\hline & 40.0 & $40.7 \pm 1.0$ & & $101.8 \pm 1.0$ & 3.1 & 3.8 \\
\hline & 80.0 & $79.9 \pm 1.0$ & & $99.9 \pm 0.5$ & 2.9 & 3.0 \\
\hline 5-MTHF & 50.0 & $49.5 \pm 3.9$ & $\mathrm{nM}$ & $99.1 \pm 3.2$ & 12.5 & 13.9 \\
\hline & 400.0 & $408.0 \pm 7.7$ & & $102.0 \pm 0.8$ & 5.7 & 5.5 \\
\hline & 800.0 & $818.2 \pm 29.8$ & & $102.3 \pm 1.5$ & 3.8 & 5.0 \\
\hline
\end{tabular}

samples spiked by serial dilution of IS solution until reaching a $S / N$ above 3 for the LOD and above 10 for the LOQ. Stable isotope-labeled compounds have the same physico-chemical properties and should therefore have the same analytical behavior as the analogue molecules (e.g., retention time, intensity). Estimated LOD and LOQ values from plasma and CSF samples spiked with IS solutions are reported in Table 2.
Linearity of the calibration We assessed whether to use solvent-based calibration curves or matrix-matched external calibration curves to accurately quantitate our 17 metabolites. Calibration curves were therefore performed in both solvent and sample matrix (plasma and CSF) using 7 calibration levels. The plasma or CSF calibration slopes differed by less than $16 \%$ from the solvent-based calibration curves. Without IS correction, these differences would have been far higher in 
Table 5 Plasma and CSF concentrations of 17 metabolites in control and AD subjects (mean $\pm \mathrm{SD})$

\begin{tabular}{|c|c|c|c|c|c|}
\hline \multirow[t]{2}{*}{ Analyte } & \multicolumn{2}{|l|}{ Plasma } & \multicolumn{2}{|l|}{ CSF } & \multirow[t]{2}{*}{ Units } \\
\hline & $\begin{array}{l}\text { Control }(n=6) \\
\text { Mean } \pm \text { SD }\end{array}$ & $\begin{array}{l}\mathrm{AD}(n=6) \\
\mathrm{Mean} \pm \mathrm{SD}\end{array}$ & $\begin{array}{l}\text { Control }(n=6) \\
\text { Mean } \pm \text { SD }\end{array}$ & $\begin{array}{l}\mathrm{AD}(n=6) \\
\mathrm{Mean} \pm \mathrm{SD}\end{array}$ & \\
\hline Homocysteic acid & $<\mathrm{LOD}$ & $<\mathrm{LOD}$ & $<\mathrm{LOD}$ & $<\mathrm{LOD}$ & $\mu \mathrm{M}$ \\
\hline Taurine & $81.5 \pm 30.9$ & $71.6 \pm 18$ & $27.8 \pm 4.7$ & $24.0 \pm 4.8$ & $\mu \mathrm{M}$ \\
\hline Serine & $79.6 \pm 15.7$ & $85.8 \pm 19.4$ & $25.4 \pm 4.8$ & $32.9 \pm 14.5$ & $\mu \mathrm{M}$ \\
\hline Total cysteine & $144.4 \pm 9.9$ & $153.2 \pm 32.9$ & $1.1 \pm 0.4$ & $2.4 \pm 2.2$ & $\mu \mathrm{M}$ \\
\hline Glycine & $250.0 \pm 112.1$ & $339.3 \pm 180.6$ & $7.8 \pm 4.4$ & $25.6 \pm 20.3$ & $\mu \mathrm{M}$ \\
\hline Total homocysteine & $5.3 \pm 1.6$ & $8.1 \pm 6.9$ & $<$ LOQ & $<\mathrm{LOQ}$ & $\mu \mathrm{M}$ \\
\hline Riboflavin & $68.2 \pm 66.6$ & $21.9 \pm 30.7$ & $6.7 \pm 5.3$ & $12.9 \pm 6.5$ & $\mathrm{nM}$ \\
\hline Methionine & $16.9 \pm 2.8$ & $16.8 \pm 3.4$ & $3.7 \pm 0.4$ & $5.3 \pm 1.6$ & $\mu \mathrm{M}$ \\
\hline Pyridoxine & $<\mathrm{LOD}$ & $<\mathrm{LOD}$ & $<\mathrm{LOD}$ & $<$ LOD & $\mathrm{nM}$ \\
\hline Cystathionine & $116.7 \pm 34.2$ & $175.7 \pm 112.3$ & $46.0 \pm 36.7$ & $42.0 \pm 32.9$ & $\mathrm{nM}$ \\
\hline SAH & $25.7 \pm 9.9$ & $40.9 \pm 9.8$ & $14.3 \pm 2.5$ & $26.1 \pm 9.4$ & $\mathrm{nM}$ \\
\hline Pyridoxamine & $<\mathrm{LOD}$ & $<\mathrm{LOD}$ & $<\mathrm{LOD}$ & $<\mathrm{LOD}$ & $\mathrm{nM}$ \\
\hline SAM & $88.5 \pm 18.1$ & $68.5 \pm 19.4$ & $191.4 \pm 31.1$ & $150.7 \pm 30.2$ & $\mathrm{nM}$ \\
\hline DMG & $<\mathrm{LOQ}$ & $<\mathrm{LOQ}$ & $<\mathrm{LOQ}$ & $<\mathrm{LOQ}$ & $\mu \mathrm{M}$ \\
\hline Betaine & $45.9 \pm 14.5$ & $45.8 \pm 17.0$ & $<\mathrm{LOD}$ & $<\mathrm{LOD}$ & $\mu \mathrm{M}$ \\
\hline Choline & $11.2 \pm 3.5$ & $11.6 \pm 3.7$ & $3.0 \pm 0.7$ & $3.5 \pm 0.5$ & $\mu \mathrm{M}$ \\
\hline 5-MTHF & $56.6 \pm 23.3$ & $191.9 \pm 363.5$ & $60.9 \pm 11.8$ & $57.4 \pm 18.8$ & $\mathrm{nM}$ \\
\hline
\end{tabular}

such complex matrices. These results show the importance of using isotopically labeled compounds as spiked IS to efficiently compensate for the matrix effect. For simplicity, only waterbased calibration curves were used during this validation to accurately quantify these analytes in plasma or CSF samples.

The linearity of the calibration curves was assessed using seven calibration levels with five replicates each. The relationship between analyte response and concentration was linear in the solvent in the range mentioned in Table S2 (see ESM). For all compounds including the additional compounds, i.e., DMG, betaine, choline, and 5-MTHF, a linear regression model was used. A weighting factor of $1 / x$ was applied for all compounds to achieve a linear model with the lowest relative errors at each level. All coefficients of determination $\left(r^{2}\right)$ were found above 0.99 . The acceptance criterion for each back-calculated standard concentration was $\pm 15 \%$ deviation from the nominal value except at the lowest level, i.e., level 1, which was set at $\pm 20 \%$. All values were found within these ranges with a maximum of 2 excluded calibration points per curve.

Trueness, precision, and recovery Values for trueness and precision are summarized in Tables 3 and 4. For plasma samples, within-run precision (repeatability $r$ ) and between-run precision (intermediate reproducibility $\mathrm{iR}$ ) $\mathrm{CV}$ values were found below $20 \%$ for the lowest spiking level and below $15 \%$ for the medium and high spiking levels, which are within the recommended limits [32]. For CSF samples, $r$ and $\mathrm{iR}$ values were found below $16 \%$ for the lowest spiking level and below $12 \%$ for the medium and high spiking levels. Recoveries were found between 90 and $108 \%$ for plasma samples and between 96 and $109 \%$ for CSF samples, which are also within the recommended limits.

Matrix effects Matrix effects may occur in any LC-MS analysis because of the presence of co-eluting compounds in the sample
Fig. 3 Plots in plasma samples. Concentrations for glycine and total homocysteine are in micromolars and for SAH and SAM in nanomolars. $P$ values are reported

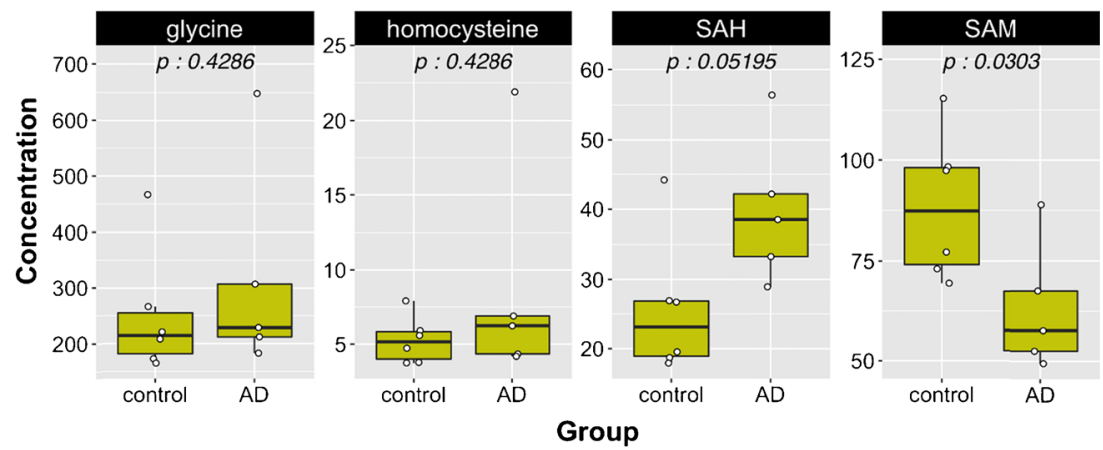


Fig. 4 Plots in CSF samples. Concentrations for glycine are in micromolars and for SAH and SAM in nanomolars. $P$ values are reported
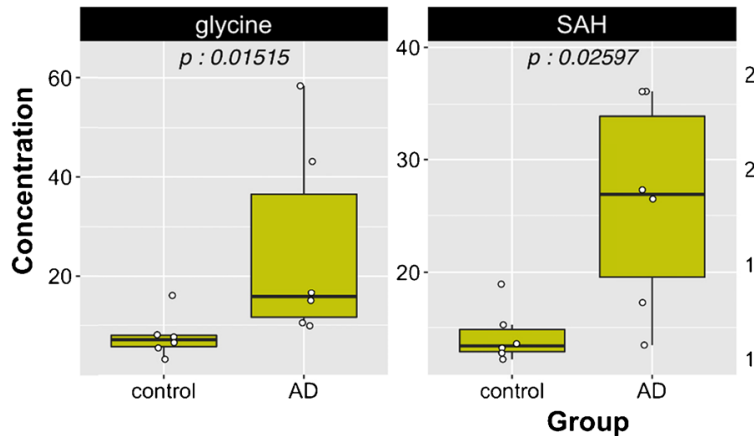

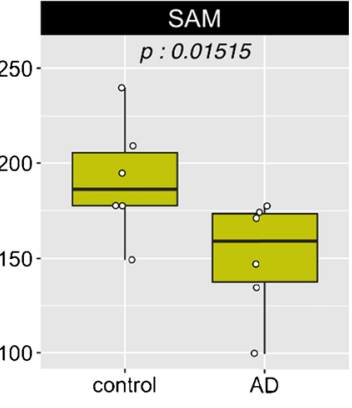

matrix, especially in complex matrices such as plasma. The use of stable isotope-labeled related compounds as IS is a wellknown efficient approach to overcome and correct matrix effects and therefore improve the method's accuracy [33]. Although stable isotope-labeled compounds were used in our method, we still evaluated ion suppression and enhancement. Besides, ion suppression may still occur when using ion pairing such as heptafluorobutyric acid. Quantitative measurements for estimating the matrix effects are presented in Table S6 in the ESM.

\section{Application to biological samples}

The homocysteine-methionine cycle is of particular importance for cognitive function and Alzheimer's disease. For instance, an imbalance of this metabolic pathway, marked by hyperhomocysteinemia and/or an altered SAM/SAH ratio, is a hallmark of memory loss and cognitive decline in elderly populations [34, 35] This loss of metabolic homeostasis may result from deficiency in metabolites and co-factors - such as vitamin $\mathrm{B}_{12}$ or folic acid - directly and indirectly involved in the methylation of homocysteine. An increased interest exists to decipher their role as potential biomarkers of neurodegeneration in Alzheimer's disease [36-38]. The robustness of our method for the sensitive detection and quantitation of the 17 metabolites and co-factors of methionine metabolism was assessed in biological materials collected from healthy donors and patients diagnosed with Alzheimer's disease. We reported in Table 5 plasma and CSF concentrations for the 17 metabolites in control and AD subjects. In blood plasma and CSF, the AD patients showed a significantly higher concentration of SAH compared to healthy subjects, but a lower circulating concentration of SAM (Figs. 3 and 4), a feature that was reported previously by other authors in several larger clinical studies using different and complementary assays [39-41]. However, despite the lack of statistical significance, our analysis also showed a trend toward a higher plasma level of homocysteine in those affected patients. In addition, a significant increase in glycine concentration in CSF was observed in AD patients, an observation that was confirmed by a similar trend in plasma samples. This metabolic pattern has also been reported previously by other authors using a targeted analytical method for amino acids [42]. Therefore, this application to real biological samples, although limited due to the number of subjects, demonstrated the importance of capturing sensitive metabolic readouts and contributed to obtain complete individual clinical phenotypes in plasma and CSF. Knowledge of the metabolites and co-factor distribution between plasma and CSF may yield information to better understand the impact of metabolic dysregulation and vitamin deficiencies/insufficiencies in the context of aging, cardiometabolic, and cognitive health research.

\section{Conclusions}

A precise evaluation of changes in the homocysteine-methionine pathway is key to understand the relation among diet, nutrition and metabolic requirements, and cardiometabolic or neurological disorders. Quantitation of elements of this pathway is often covered by several analytical methods that just cover it partially. To fill this analytical gap, we have developed a robust, highthroughput LC-MS/MS method for the quantitation of 17 key metabolites embedded into the homocysteine-methionine metabolism. The approach described here provides a novel analytical tool allowing scientists to capture in one single analysis changes in the homocysteine-methionine pathway and their co-factors in plasma and CSF samples. The proposed analytical method does this with a high level of accuracy and reproducibility, making it especially interesting for large epidemiologic studies.

Acknowledgments The authors would like to thank Jim Kaput for manuscript revision and Sebastiano Collino for his support.

Compliance with ethical standards All plasma and CSF human samples were provided by PrecisionMed, Inc. (CA, USA, protocol 8009) and collected under IRB-approved protocols.

Conflict of interest The authors declare that they have no competing interests.

Open Access This article is distributed under the terms of the Creative Commons Attribution 4.0 International License (http:// creativecommons.org/licenses/by/4.0/), which permits unrestricted use, distribution, and reproduction in any medium, provided you give appropriate credit to the original author(s) and the source, provide a link to the Creative Commons license, and indicate if changes were made. 


\section{References}

1. Rezzi S, Collino S, Goulet L, Martin FP. Metabonomic approaches to nutrient metabolism and future molecular nutrition. TrAC Trends in Analytical Chemistry. 2013;52:112-9. doi:10.1016/j. trac.2013.09.004.

2. Kaput J, Kussmann M, Radonjic M, Virgili F, Perozzi G. Human nutrition, environment, and health. Genes Nutr. 2015;10(5):489. doi:10.1007/s12263-015-0489-8

3. Collino S, Martin FP, Rezzi S. Clinical metabolomics paves the way towards future healthcare strategies. Br J Clin Pharmacol. 2012.

4. Ravaglia G, Forti P, Maioli F, et al. Homocysteine and folate as risk factors for dementia and Alzheimer disease. Am J Clin Nutr. 2005;82(3):636-43.

5. Fontecave M, Atta M, Mulliez E. S-adenosylmethionine: nothing goes to waste. Trends Biochem Sci. 2004;29(5):243-9. doi:10.1016 j.tibs.2004.03.007.

6. Collino S, Martin FP, Kochhar S, Rezzi S. Monitoring healthy metabolic trajectories with nutritional metabonomics. Nutrients. 2009;1(1):101-10. doi:10.3390/nu1010101.

7. Field MS, Shields KS, Abarinov EV, et al. Reduced MTHFD1 activity in male mice perturbs folate- and choline-dependent onecarbon metabolism as well as transsulfuration. J Nutr. 2013;143(1): 41-5. doi:10.3945/jn.112.169821.

8. Papakostas GI, Cassiello CF, Iovieno N. Folates and Sadenosylmethionine for major depressive disorder. Can $\mathrm{J}$ Psychiatry. 2012;57(7):406-13.

9. Wernimont SM, Clark AG, Stover PJ, et al. Folate network genetic variation predicts cardiovascular disease risk in non-Hispanic white males. J Nutr. 2012;142(7):1272-9. doi:10.3945/jn.111.157180.

10. Mikael LG, Pancer J, Wu Q, Rozen R. Disturbed one-carbon metabolism causing adverse reproductive outcomes in mice is associated with altered expression of apolipoprotein $\mathrm{AI}$ and inflammatory mediators PPARalpha, interferon-gamma, and interleukin-10. J Nutr. 2012;142(3):411-8. doi:10.3945/jn.111.151753.

11. Johansson M, Van Guelpen B, Vollset SE, et al. One-carbon metabolism and prostate cancer risk: prospective investigation of seven circulating B vitamins and metabolites. Cancer Epidemiol Biomarkers Prev. 2009;18(5):1538-43. doi:10.1158/1055-9965. EPI-08-1193.

12. Kirsch SH, Herrmann W, Rabagny Y, Obeid R. Quantification of acetylcholine, choline, betaine, and dimethylglycine in human plasma and urine using stable-isotope dilution ultra performance liquid chromatography-tandem mass spectrometry. J Chromatogr B Anal Technol Biomed Life Sci. 2010;878(32):3338-44. doi:10.1016/j. jchromb.2010.10.016.

13. Midttun O, Hustad S, Ueland PM. Quantitative profiling of biomarkers related to B-vitamin status, tryptophan metabolism and inflammation in human plasma by liquid chromatography/tandem mass spectrometry. Rapid Commun Mass Spectrometry: RCM. 2009;23(9):1371-9. doi:10.1002/rcm.4013.

14. Sigit JI, Hages M, Brensing KA, et al. Total plasma homocysteine and related amino acids in end-stage renal disease (ESRD) patients measured by gas chromatography-mass spectrometry-comparison with the Abbott IMx homocysteine assay and the HPLC method. Clin Chem Lab Med CCLM / FESCC. 2001;39(8):681-90. doi:10.1515/CCLM.2001.112.

15. Ueland PM, Midttun O, Windelberg A, Svardal A, Skalevik R, Hustad S. Quantitative profiling of folate and one-carbon metabolism in large-scale epidemiological studies by mass spectrometry. Clin Chem Lab Med CCLM / FESCC. 2007;45(12):1737-45. doi:10.1515/CCLM.2007.339.

16. Da Silva L, Collino S, Cominetti O, et al. High-throughput method for the quantitation of metabolites and co-factors from homocysteine-methionine cycle for nutritional status assessment. Bioanalysis. 2016;8(18):1937-49. doi:10.4155/bio-2016-0112.

17. Team RC. A language and environment for statistical computing. Vienna, Austria: R Foundation for Statistical Computing; 2016.

18. Wickham H (2009) ggplot2: elegant graphics for data analysis. Springer-Verlag, New York.

19. Obeid R. The metabolic burden of methyl donor deficiency with focus on the betaine homocysteine methyltransferase pathway. Nutrients. 2013;5(9):3481-95. doi:10.3390/nu5093481.

20. Craig SA. Betaine in human nutrition. Am J Clin Nutr. 2004;80(3): 539-49.

21. Zeisel SH, Blusztajn JK. Choline and human nutrition. Annu Rev Nutr. 1994;14:269-96.

22. Persichilli S, Gervasoni J, Iavarone F, Zuppi C, Zappacosta B. A simplified method for the determination of total homocysteine in plasma by electrospray tandem mass spectrometry. J Sep Sci. 2010;33(20):3119-24. doi:10.1002/jssc.201000399.

23. Gardner LA, Desiderio DM, Groover CJ, et al. LC-MS/MS identification of the one-carbon cycle metabolites in human plasma. Electrophoresis. 2013;34(11):1710-6. doi:10.1002/elps.201200536.

24. Hellmuth C, Koletzko B, Peissner W. Aqueous normal phase chromatography improves quantification and qualification of homocysteine, cysteine and methionine by liquid chromatography-tandem mass spectrometry. J Chromatogr B Analyt Technol Biomed Life Sci. 2011;879(1):83-9. doi:10.1016/j.jchromb.2010.11.016.

25. Rafii M, Elango R, Courtney-Martin G, House JD, Fisher L, Pencharz PB. High-throughput and simultaneous measurement of homocysteine and cysteine in human plasma and urine by liquid chromatography-electrospray tandem mass spectrometry. Anal Biochem. 2007;371(1):71-81. doi:10.1016/j.ab.2007.07.026.

26. Weaving G, Rocks BF, Iversen SA, Titheradge MA. Simultaneous quantitation of homocysteine, cysteine and methionine in plasma and urine by liquid chromatography-tandem mass spectrometry. Ann Clin Biochem. 2006;43(Pt 6):474-80.

27. Hempen $\mathrm{C}$, Wanschers $\mathrm{H}$, van der Sluijs VG. A fast liquid chromatographic tandem mass spectrometric method for the simultaneous determination of total homocysteine and methylmalonic acid. Anal Bioanal Chem. 2008;391(1):263-70. doi:10.1007/s00216008-1953-8.

28. Fu X, Xu YK, Chan P, Pattengale PK. Simple, fast, and simultaneous detection of plasma total homocysteine, methylmalonic acid, methionine, and 2-methylcitric acid using liquid chromatography and mass spectrometry (LC/MS/MS). JIMD Rep. 2013;10:69-78. doi:10.1007/8904 2012205.

29. Han JC, Han GY. A procedure for quantitative determination of tris(2-carboxyethyl)phosphine, an odorless reducing agent more stable and effective than dithiothreitol. Anal Biochem. 1994;220(1):5-10. doi:10.1006/abio.1994.1290.

30. Nolin TD, McMenamin ME, Himmelfarb J. Simultaneous determination of total homocysteine, cysteine, cysteinylglycine, and glutathione in human plasma by high-performance liquid chromatography: application to studies of oxidative stress. J Chromatogr B Analyt Technol Biomed Life Sci. 2007;852(1-2):554-61. doi:10.1016/j.jchromb.2007.02.024.

31. Gempel K, Gerbitz KD, Casetta B, Bauer MF. Rapid determination of total homocysteine in blood spots by liquid chromatographyelectrospray ionization-tandem mass spectrometry. Clin Chem. 2000;46(1):122-3.

32. US Department of Health and Human Services FaDA, Center for Drug Evaluation and Research (CDER), Center for Veterinary Medicine (CVM) (2011) Guidance for industry, bioanalytical method validation. doi:http://www.ema.europa.eu/docs/en_GB/document library/Scientific_guideline/2011/08/WC500109686.pdf

33. Luigi Silvestro ITaSRS (2013) Matrix effects in mass spectrometry combined with separation methods - comparison HPLC, GC and 
discussion on methods to control these effects. Intech Chapter 1. doi: $10.5772 / 55982$

34. Negahdar H, Hosseini SR, Parsian H, et al. Homocysteine, trace elements and oxidant/antioxidant status in mild cognitively impaired elderly persons: a cross-sectional study. Rom J Intern Med. 2015;53(4):336-42.

35. Linnebank M, Popp J, Smulders Y, et al. S-adenosylmethionine is decreased in the cerebrospinal fluid of patients with Alzheimer's disease. Neurodegener Dis. 2010;7(6):373-8. doi:10.1159/000309657.

36. Chung YC, Kruyer A, Yao Y, et al. Hyperhomocysteinemia exacerbates Alzheimer's disease pathology by way of the Abeta-fibrinogen interaction. J Thromb Haemost. 2016. doi:10.1111/jth.13340.

37. Hu Q, Teng W, Li J, Hao F, Wang N. Homocysteine and Alzheimer's disease: evidence for a causal link from Mendelian randomization. J Alzheimers Dis. 2016. doi:10.3233/JAD-150977.

38. Raszewski G, Chwedorowicz R, Chwedorowicz A, Gustaw Rothenberg K. Homocysteine, antioxidant vitamins and lipids as biomarkers of neurodegeneration in Alzheimer's disease versus non-Alzheimer's dementia. Ann Agric Environ Med. 2016;23(1): 193-6. doi:10.5604/12321966.1196878.

39. Fonteh AN, Harrington RJ, Tsai A, Liao P, Harrington MG. Free amino acid and dipeptide changes in the body fluids from Alzheimer's disease subjects. Amino Acids. 2007;32(2):213-24. doi:10.1007/s00726-006-0409-8.

40. Linnebank M, Popp J, Smulders Y, et al. S-Adenosylmethionine is decreased in the cerebrospinal fluid of patients with Alzheimer's disease. Neurodegener Dis. 2010;7(6):373-8.

41. Popp J, Lewczuk P, Linnebank M, et al. Homocysteine metabolism and cerebrospinal fluid markers for Alzheimer's disease. J Alzheimers Dis. 2009;18(4):819-28. doi:10.3233/JAD-2009-1187.

42. Jimenez-Jimenez FJ, Molina JA, Gomez P, et al. Neurotransmitter amino acids in cerebrospinal fluid of patients with Alzheimer's disease. J Neural Transm. 1998;105(2-3):269-77. doi:10.1007 /s007020050056. 